\title{
Frontal-Limbic White Matter Pathway Associations with the Serotonin Transporter Gene Promoter Region (5-HTTLPR) Polymorphism
}

\author{
Jennifer Pacheco, ${ }^{1}$ Christopher G. Beevers, ${ }^{1}$ Cristina Benavides, ${ }^{1}$ John McGeary, ${ }^{2,3}$ Eric Stice, ${ }^{1}$ and David M. Schnyer ${ }^{1}$ \\ ${ }^{1}$ Department of Psychology, The University of Texas at Austin, Austin, Texas 78712, ${ }^{2}$ Research Service, Providence Veterans Affairs Medical Center, \\ Providence, Rhode Island 02908, and ${ }^{3}$ Center for Alcohol and Addiction Studies, Brown University, Providence, Rhode Island 02912
}

\begin{abstract}
Variation in the serotonin transporter gene-linked polymorphic region (5-HTTLPR) has been associated with heightened neural activity in limbic and prefrontal regions in response to emotional stimuli. The current study examined whether the 5-HTTLPR polymorphism is also associated with alterations in microstructure of frontal-limbic white matter (WM) tracts. Thirty-seven (mean age, 20.51 years; range, 13-28) female participants were genotyped for the 5-HTTLPR polymorphism. Diffusion MRI was collected and a probabilistically defined tract of the uncinate fasciculus (UF), a WM pathway connecting the amygdala to medial and orbital prefrontal cortex, was used to generate fractional anisotropy (FA) values for participants. Regression analyses indicated a significant inverse association between number of low-expressing 5-HTTLPR alleles and FA values for the left frontal UF region, $\beta=-0.42, p=0.005$. Furthermore, there was a positive association between age and FA values for bilateral frontal regions of the UF; these effects explained 39 and $20 \%$ of the variance in FA values for left and right frontal regions, respectively. 5-HTTLPR genotype and age appear to independently influence the WM microstructure of the UF. The observed reduction in FA values among low-expressing 5-HTTLPR allele carriers may contribute to biased regulation of emotional stimuli.
\end{abstract}

\section{Introduction}

The serotonin transporter (5-HTT) regulates the reuptake of serotonin to the presynaptic neuron for recycling or degradation after serotonin has been released. It thus plays a critical role in determining the duration and intensity of serotonin communication with postsynaptic receptors and targets, such as those in limbic regions involved in the regulation of emotional information (for review, see Hariri and Holmes, 2006).

Importantly, the efficiency with which the 5-HTT returns serotonin to the presynaptic neuron appears to be influenced by the 5-HTTLPR polymorphism. A common deletion polymorphism in the promoter region of the 5-HTT gene results in two variants: a short (S) allele and a long $(\mathrm{L})$ allele. The presence of one or two $\mathrm{S}$ alleles, rather than two copies of the $\mathrm{L}$ allele, is associated with reduced transcriptional efficiency that putatively results in significant decreases $(\sim 50 \%)$ in serotonin reuptake (Caspi et al., 2003; Hu et al., 2005).

Furthermore, it has recently been determined that the long 5-HTTLPR allele has two variants (i.e., $\mathrm{L}_{\mathrm{A}}$ and $\mathrm{L}_{\mathrm{G}}$ ). In the first of two extra $20-23 \mathrm{bp}$ repeats in the $\mathrm{L}$ allele, a common single nucleotide polymorphism occurs at the sixth nucleotide (adenine

\footnotetext{
Received Feb. 20, 2009; revised March 26, 2009; accepted March 30, 2009.

This research was supported in part by U.S. Army Grant W911NK-07-2-0023 and National Institute of Mental Health Grant R01MH076897.

The authors declare no competing financial interests.

Correspondence should be addressed to Jennifer Pacheco, Department of Psychology, The University of Texas at Austin, 1 University Station A8000, Austin, TX 78712. E-mail: jpacheco@mail.utexas.edu.

D01:10.1523/JNEUROSCI.0896-09.2009

Copyright $\odot 2009$ Society for Neuroscience $\quad$ 0270-6474/09/296229-05\$15.00/0
}

to guanine; $\mathrm{A}$ to $\mathrm{G}$ ) (Wendland et al., 2006). The $\mathrm{L}_{\mathrm{G}}$ variant and the $S$ allele appear to be very similar in terms of transcriptional activity; therefore, only the $\mathrm{L}_{\mathrm{A}}$ variant is high expressing with regard to transcriptional activity (Hu et al., 2005).

This difference in how serotonin is regulated appears to impact a cortical-limbic circuit that is critical for regulating emotional information. Previous studies, among healthy participants, have shown that short 5-HTTLPR allele carriers have reduced gray matter volume in prefrontal cortex (PFC) regions (Pezawas et al., 2005). Furthermore, using functional magnetic resonance imaging (fMRI), short 5-HTTLPR allele carriers showed less functional coupling between the amygdala and perigenual anterior cingulate (pACC) (Pezawas et al., 2005), over-activation in anterior medial prefrontal regions (Heinz et al., 2005) in response to negative stimuli, and increased amygdala activation to emotional stimuli (Hariri et al., 2005). These studies indicate that the 5-HTTLPR polymorphism is associated with biased processing of emotional information, likely resulting from altered functional connectivity within a corticolimbic circuit (Munafò et al., 2008).

The rostral portion of the superior temporal gyrus and amygdala are connected to both the orbital and medial prefrontal cortex via a critical white matter (WM) tract, the uncinate fasciculus (UF) (Petrides and Pandya, 2002). The uncinate fasciculus is the largest of the three fiber tracts connecting the frontal and temporal lobes (Petrides and Pandya, 1988). Importantly, the uncinate fasciculus connects three regions involved in cognitive emotion regulation (i.e., amygdala, lateral, and medial PFC).

Recently, diffusion tensor imaging (DTI) has been used to examine the WM microstructure. Fractional anisotropy (FA) is a 
composite DTI measure corresponding to the extent of uniform directionality in the WM tracts and has been related to the degree of myelination or the number or density of fiber tracts (Beaulieu and Allen, 1994). Although the 5-HTTLPR has been associated with reduced functional coupling between areas of the PFC and amygdala (Pezawas et al., 2005), no research has examined the relation between WM architecture and 5-HTTLPR genotype. In this report, we tested the hypothesis that the 5-HTTLPR polymorphism was associated with structural differences in WM morphology among female participants. To investigate abnormalities in connectivity between critical regions involved in emotion regulation, we focused on the UF, a pathway connecting the limbic system to orbital and medial prefrontal cortex.

\section{Materials and Methods}

Participants. Thirty-seven healthy female participants (mean age, 20.5 years; range, 13-28; 18 Caucasian, seven White Hispanic, six Asian, two African American, one Native American, and three unspecified) were recruited with advertisements posted in local community centers and online. They received $\$ 65$ for their participation. Informed consent was obtained from individuals over 18 years of age; parental consent and adolescent assent was obtained for participants under the age of 18 . The Internal Review Board at the University of Texas approved this project.

Genotyping. Genomic DNA was isolated from buccal cells using a modification of published methods (Lench et al., 1988; Freeman et al., 1997). The 5-HTTLPR gene, which maps to 17q11.1-17q12, contains variable numbers of copies of a $20-23$ bp imperfect repeat sequence (Hu et al., 2006). The 14 repeat version known as the "short" or "S" version in the promoter has been associated with lower transcriptional activity and decreased expression compared with the 16-repeat version or "L" variant (D'Souza and Craig, 2008), although alternative mechanisms for this finding have been suggested (Hariri and Holmes, 2006). The primer sequences are as follows: forward, 5'-GGCGTTGCCGCTCTGAATGC-3' (fluorescently labeled), and reverse, 5'-GAGGGACTGAGCTGGACAACCAC- $3^{\prime}$.

It has recently been determined that the long 5-HTTLPR allele has two variants (i.e., $\mathrm{L}_{\mathrm{A}}$ and $\mathrm{L}_{\mathrm{G}}$ ). In the first of two extra $20-23$ bp repeats in the $\mathrm{L}$ allele, a common single nucleotide polymorphism occurs at the sixth nucleotide (adenine to guanine; A to $\mathrm{G}$ ) (Wendland et al., 2006). The $\mathrm{L}_{\mathrm{G}}$ variant and the $S$ allele appear to be very similar in terms of transcriptional activity; therefore, only the $\mathrm{L}_{\mathrm{A}}$ variant is high expressing with regard to transcriptional activity (Hu et al., 2005). To distinguish between the $S, L_{A}$, and $L_{G}$ fragments, the PCR fragment was digested with MspI according to the procedures from Wigg et al. (2006). The resulting polymorphic fragments were separated using an ABI 377 DNA Sequencer ( $\mathrm{S}: 297,127,62 \mathrm{bp} ; \mathrm{L}_{\mathrm{A}}: 340,127$, and $62 \mathrm{bp} ; \mathrm{L}_{\mathrm{G}}: 174,166,127$, and $62 \mathrm{bp})$. Allele frequencies were SS: $n=11(29.7 \%), \mathrm{SL}_{\mathrm{G}}: n=0(0 \%)$, $\mathrm{L}_{\mathrm{G}} \mathrm{L}_{\mathrm{G}}: n=0(0 \%), \mathrm{SL}_{\mathrm{A}}: n=16(43.2 \%), \mathrm{L}_{\mathrm{G}} \mathrm{L}_{\mathrm{A}}: n=2(5.4 \%)$, and $\mathrm{L}_{\mathrm{A}} \mathrm{L}_{\mathrm{A}}:$ $n=8(21.6 \%)$. Tests for Hardy-Weinberg equilibrium (HWE) were performed using the exact test of Hardy-Weinberg proportion for multiple alleles (Guo and Thompson, 1992). Proportions did not vary from $\operatorname{HWE}(p>0.05)$.

Imaging. Diffusion MRI was collected using echo planar imaging and a twice-refocused spin echo pulse sequence, optimized to minimize eddy current-induced distortions [General Electric 3T, repetition time/echo time $=12000 / 71.1, B_{0}=1000,128 \times 128$ matrix, $0.94 \times 0.94 \times 3 \mathrm{~mm}$ thick (0 mm gap) slices, 1 T2 plus $25 \mathrm{DWI}$. Forty-one slices were acquired, and the diffusion tensors and FA were calculated on a voxel-byvoxel basis using conventional reconstruction methods in FSL (FMRIB Software Library, a comprehensive library of analysis tools for fMRI, MRI, and DTI brain imaging data) (Behrens et al., 2003). FA images were registered, using a nonlinear registration, into a standard space using the FMRIB_56 template, a mean FA map derived from 56 normal young individuals registered in MNI152 standard space. The primary measure of WM was derived from regional DTI measures of FA and extracted from each participant with a pathway of interest (POI), using a probabilistically defined tract of the UF, also in standard space (Smith et al., 2006; Behrens et al., 2007), shown in Figure 1a. A similarly defined pathway of the posterior forceps was used as a control POI and analyzed in the same manner, shown in Figure $1 b$.

\section{Results}

Association between 5-HTTLPR and FA values for the UF

Linear regression analyses examined the association between number of low-expressing 5-HTTLPR alleles and FA values for the UF. The UF pathway was segmented into a frontal and a temporal portion and mean FA values were extracted from each section for each hemisphere. To control effects associated with WM microstructure development, participant age and its interaction with 5-HTTLPR genotype were also entered as covariates in each analysis.

Results indicated significant, independent genotype and age effects for FA values in the left frontal region of the UF (Table 1). As number of low-expressing alleles increased, FA values in the left frontal region of the UF decreased. Additionally, age was positively associated with FA values in this region. The additive effects of age and 5-HTTLPR genotype explained 39\% of the 
Table 1. Unstandardized and standardized betas, $t$ test, variance explained, and $F$ values for the regression models predicting $F A$ values in the left and right frontal and temporal regions of the uncinate fasciculus and left and right posterior forceps

\begin{tabular}{|c|c|c|c|c|c|c|}
\hline & \multicolumn{2}{|c|}{ Unstandardized coefficients } & \multicolumn{4}{|c|}{ Standardized coefficients } \\
\hline & B & SE & $\beta$ & $t_{(36)}$ & $R^{2}$ & $F_{(1,36)}$ \\
\hline Left frontal uncinate fasciculus & & & & & 0.39 & $7.05^{* * *}$ \\
\hline Age & 29.06 & 29.06 & 0.42 & $2.99 *$ & & \\
\hline Genotype & -165.61 & 55.11 & -0.42 & $-3.03^{*}$ & & \\
\hline Age $\times$ genotype & -4.92 & 13.82 & -0.05 & -0.36 & & \\
\hline Right frontal uncinate fasciculus & & & & & 0.21 & $2.87^{*}$ \\
\hline Age & 24.67 & 11.21 & 0.37 & $2.20^{*}$ & & \\
\hline Genotype & -20.75 & 61.80 & -0.05 & -0.34 & & \\
\hline Age $\times$ genotype & 14.61 & 15.50 & 0.16 & 0.94 & & \\
\hline Left temporal uncinate fasciculus & & & & & 0.01 & 0.20 \\
\hline Age & 6.86 & 11.78 & 0.11 & 0.58 & & \\
\hline Genotype & 17.05 & 64.97 & 0.05 & 0.26 & & \\
\hline Age $\times$ genotype & -0.68 & 16.30 & -0.01 & -0.04 & & \\
\hline Right temporal uncinate fasciculus & & & & & 0.04 & 0.50 \\
\hline Age & 5.61 & 10.19 & 0.10 & 0.55 & & \\
\hline Genotype & 28.07 & 56.20 & 0.09 & 0.50 & & \\
\hline Age $\times$ genotype & 11.40 & 14.10 & 0.15 & 0.81 & & \\
\hline Left posterior forceps & & & & & 0.04 & 0.42 \\
\hline Age & 4.305 & 16.32 & 0.05 & 0.26 & & \\
\hline Genotype & 96.56 & 90.02 & 0.19 & 1.07 & & \\
\hline Age $\times$ genotype & -5.45 & 22.58 & -0.04 & -0.24 & & \\
\hline Right posterior forceps & & & & & 0.04 & 0.47 \\
\hline Age & 2.53 & 19.48 & 0.02 & 0.13 & & \\
\hline Genotype & 83.05 & 107.43 & 0.13 & 0.77 & & \\
\hline Age $\times$ genotype & -21.71 & 26.95 & -0.15 & -0.81 & & \\
\hline
\end{tabular}

${ }^{*} p<0.05 ;{ }^{* * *} p<0.001$.
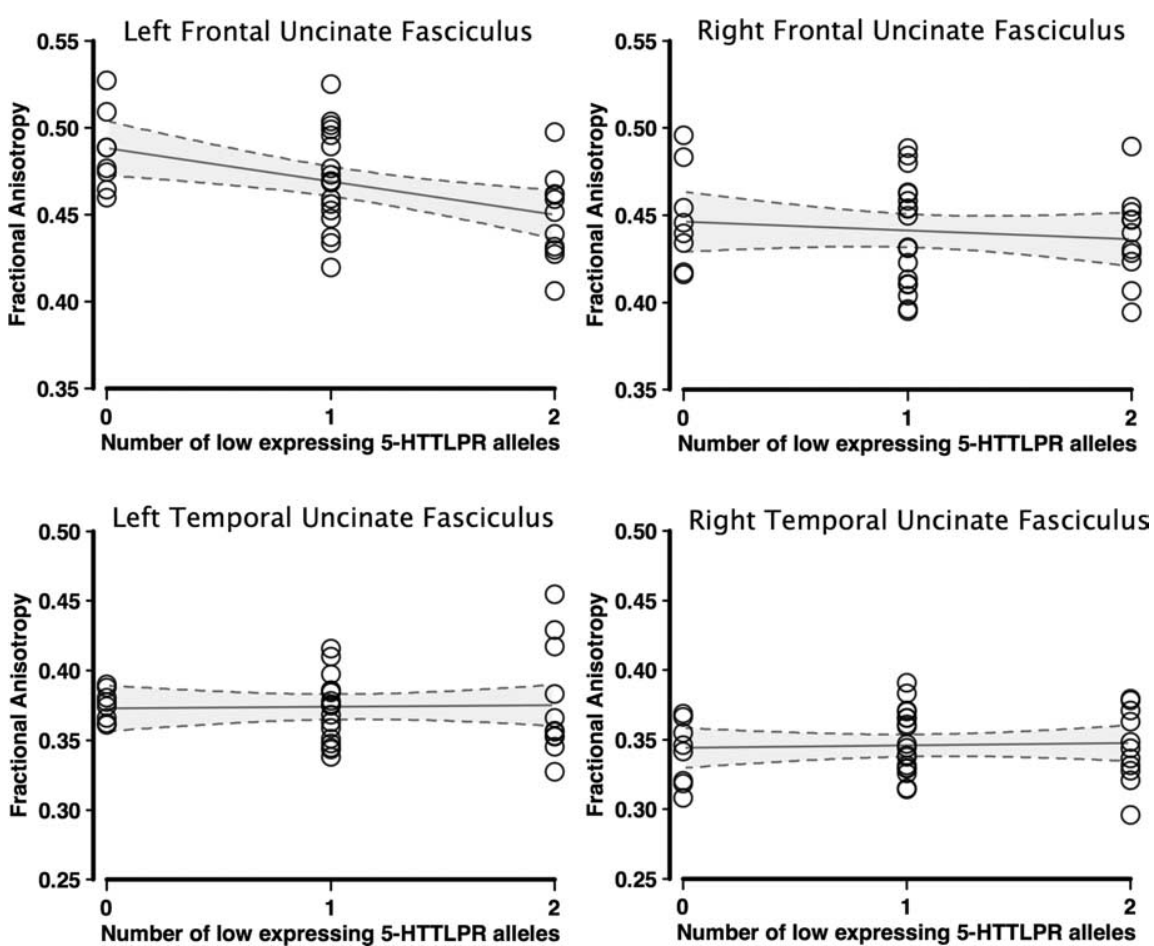

Figure 2. Scatterplots of individual FA values for frontal and temporal region of the uncinate fasciculus presented by 5-HTTLPR allele group. Regression line and $95 \%$ confidence interval is also plotted to depict the relationship between $\mathrm{FA}$ values and 5-HTTLPR for each region.

variance in FA values in the left frontal region of the UF. For the right frontal region of the UF, age was positively associated with FA values, but there was no significant association for the 5-HTTLPR polymorphism. Neither age nor 5-HTTLPR was significantly associated with FA values in the left or right temporal portions of the UF (Fig. 2, for scatter plots of individual FA values presented by 5 -HTTLPR allele group for each region of the UF).

\section{Specificity of 5-HTTLPR associations} with FA values

To examine the specificity of the group difference in the UF, FA values from the left and right hemisphere sections, excluding midline locations of the posterior forceps, were examined. This well characterized WM pathway, originating from the corpus callosum and emanating to the occipital lobe, is an ideal control region, as it is not implicated in the regulation of emotion. Analyses revealed no significant main effects or interactions for age or 5-HTTLPR genotype (Table 1).

\section{Association between FA values and ethnicity/race}

An additional regression analysis was performed to determine whether controlling for participants' ethnicity (Hispanic: yes, no) and race (Caucasian: yes, no) affected the observed association between 5-HTTLPR and left frontal FA values. [Because of the small numbers of people of non-Caucasian race, we combined them into a single race category. A chi-square test showed that there were no differences in the distribution of allele frequencies across our non-Caucasian groups $\left(\chi_{(8,15)}^{2}=3.82, p=0.87\right)$.] A regression model that included race, ethnicity, and 5-HTTLPR genotype as predictors of FA values of the left hemisphere frontal region of the UF was significant, $F_{(1,36)}=4.88, p<0.01, R^{2}=0.31$. Impor- 
tantly, the 5-HTTLPR genotype effect remained significant ( $b$, -152.18 ; SE, $61.11 ; \beta,-0.38 ; t=-2.49 ; p<0.05)$, whereas the effects for race $(b, 159.58$; SE, 94.08; $\beta,-0.27 ; t=1.70 ; p=$ $0.099)$ and ethnicity $(b,-58.09 ; \mathrm{SE}, 117.13 ; \beta,-0.08 ; t=$ $-0.50 ; p=0.623)$ were not significant.

\section{Laterality of FA values in frontal regions of UF}

To assess the apparent laterality of our findings, a repeatedmeasures ANOVA was performed, with genotype (number of low-expressing 5-HTTLPR alleles) as a between-subjects factor and hemisphere (left, right) as a within-subjects factor. There was a significant interaction between hemisphere and 5-HTTLPR allele status for FA values in the frontal regions of the uncinate fasciculus, $F_{(1,36)}=4.53, p<0.05$. This indicates that the association between the 5-HTTLPR genotype and FA values in the frontal region was significantly stronger for the left compared with right hemisphere. In contrast, there was no significant hemisphere $\times 5$-HTTLPR genotype interaction for the temporal regions of the UF, $F_{(1,36)}=0.57, p=0.57$.

\section{Discussion}

Previous research has implicated the regulation of serotonin to be important in the regulation of emotional activity, via a critical cortical-limbic circuit. Pezawas et al. (2005) found that short 5-HTTLPR allele carriers had significant reduction in gray matter volume in the pACC and the rostral anterior cingulate, regions of the prefrontal cortex previously implicated in affect regulation (Drevets, 2000), compared with long 5-HTTLPR allele homozygotes. Furthermore, using fMRI to assess relative activation of the pACC and amygdala in response to negative stimuli (e.g., angry and scared facial expressions), short 5-HTTLPR allele carriers had less functional coupling between the pACC and the amygdala. The "uncoupling" of this emotion circuit may explain why short 5-HTTLPR allele carriers have greater amygdala responses to emotional stimuli (Hariri et al., 2005).

Similarly, Heinz et al. (2005) reported that a region of the prefrontal cortex more dorsal and rostral to the pACC was overactivated in short 5-HTTLPR allele carriers than long 5-HTTLPR homozygotes when presented with emotional images. Activation of the ventromedial prefrontal cortex region was positively correlated with amygdala activation, suggesting a compensatory effort to regulate exaggerated amygdala responses of the short allele carriers. This heightened amygdala response in short 5-HTTLPR allele carriers likely applies to a variety of emotional stimuli, as the amygdala also responds to positive stimuli (Cunningham et al., 2008), as well as novel, salient, and ambiguous stimuli (Whalen, 2007).

A similar pattern of functional connectivity has been uncovered in men possessing the low-expressing allele of the monoamine oxidase A (MAOA) variable nucleotide tandem repeat (MAOA-L) (Buckholtz et al., 2008). The MAOA-L allele is associated with increased levels of 5-HT, and the functional network of ventromedial prefrontal cortex, rostral cingulate, and amygdala has been shown to be both functionally and structurally compromised during an emotional arousal task among MAOA-L allele carriers. Together, these results suggest that alterations in 5-HT concentrations during development may affect critical pathways for emotion regulation by altering white matter microarchitecture (Buckholtz and Meyer-Lindenberg, 2008). This is further supported by the critical role 5-HT plays in the modulation of axonal guidance during development (Bonnin et al., 2007).

These studies indicate that the 5-HTTLPR polymorphism is associated with biased processing of emotional information, likely resulting from altered functional connectivity within a corticolimbic circuit (Munafò et al., 2008). Critical components of this circuit include the amygdala, subgenual anterior cingulate cortex, and the prefrontal cortex, which are involved in the experience, expression, and regulation of emotion. Therefore, individual differences in the microarchitecture of white matter fiber tracts that connect cortical and limbic regions could strongly affect the ability to cognitively regulate emotional information. Despite several findings indicating that the 5-HTTLPR is associated with reduced functional coupling between areas of the prefrontal cortex and amygdala, no previous research has directly tested this possibility by examining whether the 5-HTTLPR is associated with individual differences in the WM pathways that connect these regions.

In this study, we have documented a strong inverse association between number of low-expressing 5-HTTLPR alleles and the WM microarchitecture of a frontal-limbic circuit that plays a critical role in the regulation of emotional reactivity. As number of low-expressing 5-HTTLPR alleles increased, FA values in the left frontal region of the uncinate fasciculus decreased. Lower FA values in the uncinate fasciculus, a critical WM tract that connects regions of the prefrontal cortex to the amygdala, may contribute to the heightened amygdala reactivity and poorer functional coupling between the prefrontal cortex and amygdala (Pezawas et al., 2005) observed among carriers of the lowexpressing 5-HTTLPR allele. Future work that assesses whether WM microarchitecture mediates the association between 5-HTTLPR polymorphism and neural responses to emotional stimuli is needed to confirm this hypothesis.

Importantly, reduced FA values in the left frontal region of the uncinate fasciculus were observed among a sample of healthy, nondepressed, nonmedicated women. Therefore, the results from the current study are likely associated with vulnerability to depression (Caspi et al., 2003), rather than a symptomatic outcome of altered mood state. Furthermore, it is intriguing that FA differences were specific to the UF in the left frontal regions. This is consistent with previous research indicating altered activity in left prefrontal cortex is associated with vulnerability to depression (Davidson et al., 2002), although additional research is needed to determine whether the laterality of these effects are replicated in a larger sample.

We also documented a strong association between age and FA values specifically in the bilateral frontal regions of the uncinate fasciculus. This finding corresponds with work showing FA values in prefrontal regions (including those associated with attention, motor skills, and cognitive ability) increase with age as prefrontal myelination develops (Barnea-Goraly et al., 2005). Given the high rates of depression observed among adolescents, particularly girls (Hankin et al., 1998), alterations in the development of the uncinate fasciculus may play an important role in the onset of depression. Consistent with this possibility, the 5-HTTLPR polymorphism appears to be an important predictor of depression vulnerability among adolescent girls (Eley et al., 2004).

Several limitations of this study should be noted. First, our sample size was modest and exclusively female: a replication study with a larger sample of men and women is needed. Third variable explanations, such as the possibility that the 5-HTTLPR promoter polymorphism is in linkage disequilibrium with another functional genetic marker or the possibility of population stratification, should also be considered as explanations for the observed effects. Our sample was racially heterogeneous, but we found no differences in allele frequencies for the minority groups, 
so they were collapsed together for analysis. Although we found no evidence that race or ethnicity predicted FA values in any of the regions we examined, future work with larger samples should consider the races separately, as there are known allele frequency differences for African and Asian races, or consider using genomic controls.

Finally, it will be critically important for future work to determine whether impairments in the development of the uncinate fasciculus (and other white matter pathways involved in the regulation of emotion) predict future onset of depression. Consistent with this possibility, this is the first demonstration of an association between the 5-HTTLPR polymorphism, a gene that has been linked with depression vulnerability (Caspi et al., 2003), and the white matter morphology of the uncinate fasciculus.

\section{References}

Barnea-Goraly N, Menon V, Eckert M, Tamm L, Bammer R, Karchemskiy A, Dant CC, Reiss AL (2005) White matter development during childhood and adolescence: a cross-sectional diffusion tensor imaging study. Cereb Cortex 15:1848-1854.

Beaulieu C, Allen PS (1994) Determinants of anisotropic water diffusion in nerves. Magn Reson Med 31:394-400.

Behrens TE, Woolrich MW, Jenkinson M, Johansen-Berg H, Nunes RG, Clare S, Matthews PM, Brady JM, Smith SM (2003) Characterization and propagation of uncertainty in diffusion-weighted MR imaging. Magn Reson Med 50:1077-1088.

Behrens TE, Berg HJ, Jbabdi S, Rushworth MF, Woolrich MW (2007) Probabilistic diffusion tractography with multiple fibre orientations: What can we gain? Neuroimage 34:144-155.

Bonnin A, Torii M, Wang L, Rakic P, Levitt P (2007) Serotonin modulates the response of embryonic thalamocortical axons to netrin-1. Nat Neurosci 10:588-597.

Buckholtz JW, Meyer-Lindenberg A (2008) MAOA and the neurogenetic architecture of human aggression. Trends Neurosci 31:120-129.

Buckholtz JW, Callicott JH, Kolachana B, Hariri AR, Goldberg TE, Genderson M, Egan MF, Mattay VS, Weinberger DR, Meyer-Lindenberg A (2008) Genetic variation in MAOA modulates ventromedial prefrontal circuitry mediating individual differences in human personality. Mol Psychiatry 13:313-324.

Caspi A, Sugden K, Moffitt TE, Taylor A, Craig IW, Harrington H, McClay J, Mill J, Martin J, Braithwaite A, Poulton R (2003) Influence of life stress on depression: moderation by a polymorphism in the 5-HTT gene. Science 301:386-389.

Cunningham WA, Van Bavel JJ, Johnsen IR (2008) Affective flexibility: evaluative processing goals shape amygdala activity. Psychological Science 19:152-160.

Davidson RJ, Pizzagalli D, Nitschke JB, Putnam K (2002) Depression: perspectives from affective neuroscience. Annu Rev Psychol 53:545-574.

Drevets WC (2000) Neuroimaging studies of mood disorders. Biol Psychiatry 48:813-829.

D'Souza UM, Craig IW (2008) Functional genetic polymorphisms in serotonin and dopamine gene systems and their significance in behavioural disorders. Prog Brain Res 172:73-98.

Eley TC, Sugden K, Corsico A, Gregory AM, Sham P, McGuffin P, Plomin R,
Craig IW (2004) Gene-environment interaction analysis of serotonin system markers with adolescent depression. Mol Psychiatry 9:908-915.

Freeman B, Powell J, Ball D, Hill L, Craig I, Plomin R (1997) DNA by mail: An inexpensive and noninvasive method for collecting DNA samples from widely dispersed populations. Behav Genet 27:251-257.

Guo SW, Thompson EA (1992) Performing the exact test of HardyWeinberg proportion for multiple alleles. Biometrics 48:361-372.

Hankin BL, Abramson LY, Moffitt TE, Silva PA, McGee R, Angell KE (1998) Development of depression from preadolescence to young adulthood: emerging gender differences in a 10-year longitudinal study. J Abnorm Psychol 107:128-140.

Hariri AR, Holmes A (2006) Genetics of emotional regulation: the role of serotonin transporter in neural function. Trends Cog Sci 10:182-191.

Hariri AR, Drabant EM, Munoz KE, Kolachana BS, Mattay VS, Egan MF, Weinberger DR (2005) A susceptibility gene for affective disorders and the response of the human amygdala. Arch Gen Psychiatry 62:146-152.

Heinz A, Braus DF, Smolka MN, Wrase J, Puls I, Hermann D, Klein S, Grüsser SM, Flor H, Schumann G, Mann K, Büchel C (2005) Amygdalaprefrontal coupling depends on a genetic variation of the serotonin transporter. Nat Neurosci 8:20-21.

Hu X, Oroszi G, Chun J, Smith TL, Goldman D, Schuckit MA (2005) An expanded evaluation of the relationship of four alleles to the level of response to alcohol and alcholism risk. Alcohol Clin Exp Res 29:8-16.

Hu XZ, Lipsky RH, Zhu G, Akhtar LA, Taubman J, Greenberg BD, Xu K, Arnold PD, Richter MA, Kennedy JL, Murphy DL, Goldman D (2006) Serotonin transporter promoter gain-of-function genotypes are linked to obsessive-compulsive disorder. Am J Hum Genet 78:815-826.

Lench N, Stanier P, Williamson R (1988) Simple non-invasive method to obtain DNA for gene analysis. Lancet 1(8599):1356-1358.

Munafò MR, Brown SM, Hariri AR (2008) Serotonin transporter (5HTTLPR) genotype and amygdala activation: a meta-analysis. Biol Psychiatry 63:852-857.

Petrides M, Pandya DN (1988) Association fiber pathways to the frontal cortex from the superior temporal region in the rhesus monkey. J Comp Neurol 273:52-66.

Petrides M, Pandya DN (2002) Association pathways of the prefrontal cortex. In: Principles of frontal lobe function (Stuss DT, Knight RT, eds). New York: Oxford UP.

Pezawas L, Meyer-Lindenberg A, Drabant EM, Verchinski BA, Munoz KE, Kolachana BS, Egan MF, Mattay VS, Hariri AR, Weinberger DR (2005) 5-HTTLPR polymorphism impacts human cingulate-amygdala interactions: a genetic susceptibility mechanism for depression. Nat Neurosci 8:828-834.

Smith SM, Jenkinson M, Johansen-Berg H, Rueckert D, Nichols TE, Mackay CE, Watkins KE, Ciccarelli O, Cader MZ, Matthews PM, Behrens TE (2006) Tract-based spatial statistics: voxelwise analysis of multi-subject diffusion data. Neuroimage 31:1487-1505.

Wendland JR, Martin BJ, Kruse MR, Lesch KP, Murphy DL (2006) Simultaneous genotyping of four functional loci of human SLC6A4, with a reappraisal of 5-HTTLPR and rs25531. Mol Psychiatry 11:224-226.

Whalen PJ (2007) The uncertainty of it all. Trends Cogn Sci 11:499-500.

Wigg KG, Takhar A, Ickowicz A, Tannock R, Kennedy JL, Pathare T, Malone M, Schachar R, Barr CL (2006) Gene for the serotonin transporter and ADHD: no association with two functional polymorphisms. Am J Med Genet B Neuropsychiatr Genet 141B:566-570. 